\title{
The Genetics of IgA Nephropathy: An Overview from Western Countries
}

\author{
John Feehally Jonathan Barratt \\ The John Walls Renal Unit, University Hospitals of Leicester, and Department of Infection, Immunity and \\ Inflammation, University of Leicester, Leicester, UK
}

\section{Key Words}

IgA nephropathy - Genome-wide association study .

Candidate genes - Single nucleotide polymorphisms . HLA

\begin{abstract}
Background: IgA nephropathy (IgAN) is the commonest primary glomerulonephritis worldwide and a significant cause of chronic kidney disease and end-stage renal disease. It is widely accepted that genetic factors play a role in the pathogenesis of IgAN. However, the identity of these genetic factors remains uncertain. Summary: Critical to all genetic studies is a precise phenotypic definition of the disease. It is well recognised that IgAN displays striking phenotypic variation, raising the possibility that it may not be a single disease and it may not be the same disease in different parts of the world. In this review, we discuss the challenges that this phenotypic variation poses to interpreting genetic data and the current evidence for specific gene involvement in IgAN, focusing particularly on data from European IgAN cohorts. Key Messages: With advances in genetic techniques, in particular next-generation sequencing, and an increased understanding of the importance of copy number variations, epigenetics and transcriptomics, it is likely that we will gain a greater understanding of the genetic basis for IgAN. However, due to the lack of consistency in epidemiological clinicopathological studies both within and between continents,
\end{abstract}

this will only be achieved if we are able to more precisely phenotype IgAN populations. Facts from East and West: The reported prevalence of IgAN is higher in Asia than in Europe and North America. However, differences in use of biopsy for the diagnosis of IgAN should be taken into account in analysing data from both East and West. In Europe, IgAN affects men more frequently than women; this is not the case in Asia. Familial IgAN has been more frequently reported in Europe than in Asia. Within Europe, familial IgAN is more evident in southern than in northern populations. Changes in the pattern of serum IgA 1 O-glycosylation is a common finding in IgAN patients in the East and West. SNPs within the gene coding for the enzyme C1GALT1 have been reported in Chinese and European patients. However, there is no evidence for a role of gene polymorphism of the C1GALT1 chaperone cosmc in Europeans. Genetic variants in the HLA gene family have been observed in populations from the East and West. Associations between IgAN and variants of the TAP1/PSMB and DEFA genes were observed in Asian but not in Western patients. Association with the angiotensinconverting enzyme gene was seen only in Asian patients.

(C) 2015 S. Karger AG, Base

For an overview of the genetics of IgA nephropathy in China, see Zhu and Zhang, Kidney Dis 2015, DOI:10.1159/000381740, www. karger.com/doi/10.1159/000381740.

\section{KARGER 125}

(c) 2015 S. Karger AG, Base

2296-9381/15/0011-0033\$39.50/0
John Feehally

The John Walls Renal Unit, Leicester General Hospital

Gwendolen Road

Leicester LE5 4PW (UK)

www.karger.com/kdd
E-Mail jf27@le.ac.uk 


\section{Introduction}

Soon after the first description of IgA nephropathy (IgAN) in 1968, interest developed in the possibility that genetic factors played a role in the pathogenesis of this common glomerular disease, and data addressing this issue have been published since the 1970s.

In this review, we will discuss the rather slow progress made in the analysis of the genetics of IgAN over the last 40 years, with particular focus on data derived from populations of European origin. We will consider some of the reasons why that progress has been slower than initially hoped, we will review critically the strengths and limitations of the currently available data, and make recommendations for the future directions of these studies.

\section{Progress in Genetic Techniques}

A succession of genetic techniques has been available for the study of the genetic associations with disease over the last 40 years. The strengths and limitations of each successive new technique have not always been well understood by nephrologists who are not trained in this field. As a consequence, there has typically been a cycle of overoptimism in the strength of each technique, followed by overinterpretation of available data and false conclusions, followed by disappointment and negativity. Realism about strengths and limitations of the methods and the consequent data is required.

\section{Challenges in the Genetic Analysis of IgAN}

Genetic techniques which have been applied in the study of IgAN use either a family-based approach or a population-based approach. Family-based approaches have mainly used family aggregation studies or wholegenome linkage. Population-based approaches have mainly used candidate gene association studies and, more recently, genome-wide case control association studies (GWAS). It is also important to appreciate that that there are features of IgAN that make it harder for genetic analysis to be informative.

\section{Is IgAN a Single Disease?}

The definition of IgAN is apparently straightforward: the single finding of diffuse mesangial IgA deposition. But the phenotype variations are striking. These include variations in clinical presentation and progression, varia- tions in renal histopathology (although defined by mesangial $\operatorname{IgA}$, a wide range of glomerular and interstitial injury can ensue), variations in transplant recurrence, and variations in apparent geographical incidence [1]. While there are unifying explanations which can draw these together, it must still be accepted that there is no proof that the entity we currently call IgAN is a single 'disease' (using the term 'disease' in its conventional sense of a single entity sharing aetiological factors and pathogenic processes). There is also no proof that IgAN is the same 'disease' in all parts of the world. This uncertainty has implications for our understanding of the genetic basis for the disease.

\section{The Problem of Phenotyping}

Any genetic analysis investigating associations between inherited factors and disease risk is built on the presumption that the disease, in this case IgAN, can be identified with certainty in all study subjects, and equally importantly, that the absence of IgAN can be identified with confidence in subjects included in a control study population. Since the definition of IgAN requires the identification of mesangial IgA deposits, all subjects with IgAN will have undergone renal biopsy, and disease identification would appear to be straightforward. Inevitably all such patients will have had clinically identifiable kidney disease at the time of renal biopsy, although in many it may have been asymptomatic, identified for example by routine urine testing. However it is also necessary to be confident that cohorts designated as controls are indeed disease free. It is known that a significant proportion of the general population have mesangial IgA deposition (identified in autopsies [2] and also in living kidney donors [3]) yet have never had clinically detected kidney disease. We know that clinically mild IgAN may become clinically undetectable during follow-up [4]; it is therefore impossible in the absence of a biopsy to know whether individuals identified as healthy controls for these studies are indeed free of IgA deposition. Whether mesangial IgA in the absence of clinically evident kidney disease can be considered as IgAN is an unresolved semantic discussion, but it has to be recognised that such individuals have probably been included in control cohorts in all genetic studies in IgAN.

The proper identification of those with IgAN in family studies presents additional important challenges. When IgAN has been identified by renal biopsy in one family member it is common that the presence or absence of IgAN in other family members is deduced without a biopsy from the presence or absence of any urine abnormality (haematuria with or without proteinuria) or other 
features of kidney disease. This is an unsatisfactory approach to ascertainment since there are other glomerular causes of haematuria. In particular, thin membrane nephropathy (TMN), which also typically presents with non-visible haematuria and can co-segregate with IgAN in some families [5]. It is perfectly possible that among those families identified as having IgAN on the basis of urinalysis alone there will be individuals with TMN but without IgAN. Furthermore, not all family studies have included electron microscopy (EM) in the analysis of those who have been biopsied, meaning it is not possible to define with confidence the contribution of co-existing TMN (which can only be identified by EM). Since TMN is known to be associated with modifications in basement membrane collagen genes, an accurate understanding of the distribution of TMN within any IgAN cohort is required for informed analysis of genetic associations.

The assembly of cohorts of IgAN for genetic analysis is typically achieved by case ascertainment through renal biopsy or clinical databases searched for IgAN. To assemble sufficient cases, some of those included will often have been biopsied several years before study recruitment. Cohorts will include those with ESRD at one extreme, and at the other extreme those whose only clinical manifestation is minor urine abnormality (how minor will depend on each nephrologist's attitude to renal biopsy in such patients). Patients included in published genetic analyses of IgAN therefore include those with IgAN who have progressed to ESRD, and have done so at very different rates, those who have no evidence of disease progression at recruitment, and never will progress, as well as those who have not progressed yet but will do so in the future. If, as seems probable, genetic factors are an important influence on the risk and rate of progressive renal failure in IgAN, the mixing of variable progression risk in any cohort may be a significant confounder for genetic analysis. No published genetic study in European patients or any other population in IgAN has yet included sufficient phenotypic analysis to identify progressors and non-progressors, and this is a challenge given the slow rate of progression which is characteristic of IgAN.

\section{Are ‘Genetic Factors' Important in IgAN?}

Given these ascertainment and recruitment issues, what is the current evidence that IgAN has a genetic basis? There are several strands of evidence which build the case that genetic factors are indeed an important influence on pathogenesis of IgAN.

The Genetics of IgA Nephropathy in Western Countries
Varying Prevalence of IgAN in Different Ethnic

Groups

The original descriptions of IgAN were all in patients of European origin, initially in France [6] then in Australia, United States, and soon followed by studies from UK, Germany, Italy and Spain. IgAN became accepted as one of the commonest patterns of glomerular disease especially in younger males. By the 1980s, evidence emerged that IgAN may be even more common in North and East Asians than in European populations and is epidemiologically distinct, most notably in its equal distribution between genders in Asians [7], contrasted with the marked male dominance in Europeans. Caution may therefore be required when considering the relevance of genetic observations in Asians compared to European populations. And attention must be paid to the relevance of differences in ethnicity within the large cohorts used in GWAS.

More recently, the geographical variations in IgAN have been more carefully defined and show a west-to-east gradient from Africa, both in disease incidence and in distribution of currently understood risk alleles [8]. However, it has also been known for some time that even within Europe there are important variations, between north and south. IgAN appears to be more common in Mediterranean Europe [9]. It has always been debated whether these and other apparent differences in prevalence represent true differences in incidence or may be influenced by variations in the use of renal biopsy. Data from Scotland favour the latter, showing that in two centres only $100 \mathrm{~km}$ apart, there was a 70\% difference in the incidence of IgAN which was associated with a marked increase in the number of renal biopsies per year [10], implying that increasing the range of people subjected to renal biopsy, presumably typically with minor urine abnormalities, will increase the identification of IgAN. Data from other registries across Europe also show an increase in the reported incidence of IgAN as the biopsy rate increases.

\section{Increased Risk of IgAN in Relatives of Patients}

A second reason for considering genetic factors to be of importance in IgAN is the evidence that there is an increased risk of IgAN in the relatives of probands, particularly in reports from Southern Europe. The prevalence of familial IgAN and of urine abnormalities among family members of probands was first described in Italy: nonvisible haematuria was identified by systematic screening in $24 \%$ of relatives of IgAN patients [11]. The same group went on to quantify a 16 -fold increased risk of IgAN in first-degree relatives [12]. This has not been confirmed in studies from northern Europe. 
Table 1. Reported kindreds with familial IgAN

\begin{tabular}{lllll}
\hline Country & $\begin{array}{l}\text { First author [ref.], } \\
\text { year }\end{array}$ & $\begin{array}{l}\text { Locus associated } \\
\text { with IgAN }\end{array}$ & Designation & Comments \\
\hline USA & Gharavi [13], 2000 & 6q22-23 & IGAN1 & \\
Italy & Bisceglia [14], 2006 & $\begin{array}{l}\text { qq26-31 } \\
\text { IGAN2 }\end{array}$ & IGAN3 & Includes COL4A3, 4A4 \\
Canada & Paterson [15], 2007 & 2q36 & & No linkage to 6q22-23, 2q36, 4q26-31 \\
Lebanon & Karnib [16], 2007 & - & & \\
\hline
\end{tabular}

\section{Mendelian Transmission in (Rare) Kindreds}

Four kindreds have been described in which IgAN is extensively represented in patterns suggesting Mendelian inheritance. In each case, the pattern is suggestive of autosomal dominant inheritance with incomplete penetrance.

These pedigrees have been extensively studied with the presumption that their genetics may be highly informative about the genetics of 'typical' sporadic IgAN. Three of these four kindreds are people of European origin - in the USA [13], in Italy [14], and in Canada [15]. The fourth is reported from Lebanon [16]. The linkages identified in these pedigrees are shown in table 1 .

The single most striking finding is that genetic linkage differs in each of the four kindreds, arguing strongly against any generalisability of these findings to sporadic IgAN. Furthermore, studying the three novel loci (designated IGAN1, IGAN2, IGAN3) has identified no likely candidate genes. Only the 2q36 locus identified in the Canadian pedigree genes is potentially informative since it contains COL4A3 and COL4A4 genes coding for basement membrane collagen, mutations of which are associated with TMN [17].

All of these pedigrees are beset by the ascertainment uncertainties discussed above, since abnormal urinalysis has in a considerable number of cases been regarded as sufficient evidence of IgAN, and if there has been a renal biopsy, EM is infrequently available so that the prevalence of coincidental TMN (defined by EM appearances) cannot be determined.

\section{Evidence from Population-Based Approaches}

Candidate Gene Association Studies

The yield of data providing robust insights from these studies has been disappointingly small. The candidate gene approach relies on the identification of candidate genes with biological plausibility for relevance to pathogenesis and the availability of identifiable markers of genetic variations with the candidate gene. A variety of techniques have been used, most commonly RFLP (restriction fragment length polymorphism) analysis. The first candidate gene association studies from the late 1970s were carried out in European patients, and the first Asian study was published in 1984 . Since then, more than 150 such studies of candidate genes in IgAN have now been published, almost all in cohorts of fewer than 150 patients [18]. It is notable that more than $70 \%$ of the published reports identify a positive association between a candidate gene and IgAN, yet the reproducibility of many positive findings in subsequent studies is low. These observations are suggestive of publication bias, and also may in part be explained by the risk of false-positive findings in small study populations when data are exposed to multiple statistical testing. It is now thought that the inconsistency of these findings is explained in part by the haplotype block structure of the human genome [19].

\section{Genome-Wide Association Studies}

Evidence for genetic factors in IgAN comes also from genome-wide association case control studies (GWAS). GWAS have dominated the approach to genetic disease susceptibility across a broad swathe of medicine over the last decade [20]. The principle is to genotype with a large number of markers, typically at least 600,000 SNPs (single nucleotide polymorphisms), across the genome in cases and controls. GWAS will identify common alleles in a population that are present at a different frequency in people with disease, based on an assumption that the study population is a good representation of the clinical population affected by the disease being studied. The strength of GWAS is its lack of bias; there are no a priori genomic assumptions. The weakness of GWAS is that areas of the genome are identified by SNPs which are associated with disease, but this does not precisely define the genes involved. This is an important principle since the discussion of disease-associated loci identified by GWAS is too often taken to imply that the genes themselves have been proven 
to be disease modifiers. GWAS also have potential for false signal: well-matched controls are needed, and great precision is required in the techniques used for DNA isolation and genotyping. GWAS are also sensitive to population stratification. Care is needed to confirm the uniformity of ancestry in any populations studied. For example, the first reported GWAS in IgAN was a UK study of 914 patients, relatives and controls, all of whom self-identified as being of White European ethnicity. Yet, principal component analysis identified $10 \%$ of the study cohort who had to be excluded because of clear evidence of mixed ethnicity involving African or Asian population genetic markers [21].

Three independent GWAS in different populations with IgAN have been reported [21-23], and recently a fourth meta-analysis incorporating the populations from all three previous GWAS and including additional populations from both Europe and Asia [24].

The populations studied and main findings in the four published GWAS are summarised in table 2. The increasing size of the populations studied has not unexpectedly made successive GWAS more informative, providing encouraging reproducibility of significant associations, and revealing increasing numbers of risk alleles with lesser degrees of statistical significance as the study populations have increased.

While European populations have been included in three of the four published GWAS, the populations studied have been dominated by Chinese cohorts. Only $43 \%$ of patients in the GWAS discovery cohorts are European, and only $38 \%$ of the replication cohorts. Furthermore they have originated from different parts of Europe. In the first GWAS, all subjects were resident in the UK, in subsequent studies cohorts from northern and southern Europe have been included, but in only two of the GWAS was there a cohort from the USA. None have yet studied people of European origin living elsewhere in the world, for example in Australasia or Latin America. While populations from different parts of Europe may have relative genetic homogeneity, environmental variability may confer important variations not identified by genomic study. For example, diet and other environmental differences may modify phenotype independently of genetic processes; or such differences may have epigenetic effects (see below) modifying gene expression.

While successive GWAS of increasing size have continued to identify new risk alleles which are hypothesis-generating, it is perhaps disappointing that a smaller number of higher risk alleles has not emerged. This may in part be explained by the issues of phenotypic definition discussed earlier. There is a contrast with the analytical power avail-

The Genetics of IgA Nephropathy in Western Countries able when there is a disease definition based on a very tightly defined clinicopathological phenotype such as membranous nephropathy, in which a remarkably small cohort of patients, all of White European origin, yielded in GWAS a very strong associative signal with SNPs in only two areas of the genome: one in the HLA region on chromosome 6 , and the other on chromosome 1 in the region where the commonest autoantigen in membranous nephropathy, phospholipase A2 receptor, is coded. It remains to be seen if the power of GWAS will increase substantially in IgAN when we become better skilled at refining and restricting its diagnostic and phenotypic categories.

The strongest associations in the GWAS are with areas of the genome associated with a number of immune-mediated disorders, notably regions coding for HLA and complement. In all four published IgAN GWAS, the most strongly associated risk alleles are multiple alleles within the HLA region at chromosome 6p21 (table 2). Another risk allele at chromosome 1q32 points to a role for complement-regulatory proteins. Despite the finding of C3 in many biopsies in IgAN, there were few early studies of the role of complement in IgAN, in part because IgA was regarded as a poor activator of complement. However, interest was refreshed by evidence that engagement of the mannose-binding lectin pathway of complement activation was implicated in glomerular injury in IgAN [25]. Now, analysis from GWAS has identified associations between IgAN SNPs in regions coding complement regulatory proteins, most notably CFRH1/3. Whether genetic variations in factor $\mathrm{H}$ are indeed implicated in variations in pathogenic processes in IgAN requires further study.

The identification of an increased number of other risk alleles (table 2) gives the opportunity for clustering of these alleles to be sought using the National Human Genomes Research Institute GWAS catalogue, and by comparison with the KEGG (Kyoto Encyclopaedia of Genes and Genomes) pathways. This has shown that there is enrichment of the IgAN GWAS for SNPs implicated in autoimmune or inflammatory traits, and furthermore the majority of loci associated with IgAN encode proteins implicated in the maintenance of the intestinal barrier and regulation of mucosal immune response to pathogens [24]. These interesting observations are hypothesisgenerating but should not yet be taken as proof that genetic variations in these pathways are involved the pathogenesis of IgAN.

Finally, there is an increasing interest in copy number variations ( $\mathrm{CNV}$, loosely defined as a deletion, duplication or inversion of a DNA sequence longer than $1 \mathrm{~kb}$ ) as a source of genetic variation because CNV could in prin- 
Table 2. GWAS in IgAN: populations studied and significant associations

\begin{tabular}{|c|c|c|c|c|}
\hline & Feehally [21], 2010 & Gharavi [22], 2011 & $\mathrm{Yu}[23], 2012$ & Kiryluk [24], 2014 \\
\hline $\begin{array}{l}\text { Discovery } \\
\text { cohort }\end{array}$ & 431 European & 1,194 Chinese ${ }^{a}$ & 1,434 Chinese & $\begin{array}{l}\text { 1,194 Chinese }{ }^{\mathrm{a}} \\
\text { 1,553 European (Italy, USA, France) }\end{array}$ \\
\hline $\begin{array}{l}\text { Replication } \\
\text { cohort }\end{array}$ & - & $\begin{array}{l}712 \text { Chinese } \\
\text { 1,238 European (Italy, USA) }\end{array}$ & 2,703 Chinese & $\begin{array}{l}\text { 2,046 Chinese } \\
445 \text { Japanese } \\
\text { 2,420 European (UK, France, } \\
\text { Germany, Italy, Czech Republic, } \\
\text { Hungary, Turkey, Poland) }\end{array}$ \\
\hline \multirow{9}{*}{$\begin{array}{l}\text { Significant } \\
\text { associations }\end{array}$} & 6p21 HLA (multiple) & 6p21 HLA (multiple) & 6p21 HLA (multiple) & 6p21 HLA (multiple) \\
\hline & & 6p21 TAP1/PSMB8 & - & 6p21 TAP1/PSMB8 (Asian only) \\
\hline & - & 1q32 CFHR1/3 & - & 1q32 CFHR $1 / 3$ \\
\hline & - & 22q12 HORMAD2 & - & 22q12 HORMAD2 \\
\hline & - & - & 17p13 TNFSF13 & 17p13 TNFSF13 \\
\hline & - & - & 8p23 DEFA & 8p23 DEFA (Asian only) \\
\hline & & & & 6p11 ITGAM-ITGAX \\
\hline & & & & 1 p13 VAV3 \\
\hline & & & & 9q34 CARD9 \\
\hline
\end{tabular}

The studies are listed as first author [ref.], year.

a The Chinese discovery cohort was the same in these studies.

ciple account for a significant variation in disease risk [26]. Through innovations in the design and analysis of SNP arrays and by progress in determining the genomic locations and population-genetic properties of the CNVs that segregate in the human population, it is increasingly possible to study CNV as an extension of GWAS. Such studies are beginning to result in discoveries of both de novo and inherited $\mathrm{CNV}$ that are associated with risk of common disease [27]. A recent report in Italian patients with familial IgAN identified a copy number variable region at 3p21.1 that influenced toll-like receptor-9 (TLR9) expression levels [28]. Low copy number was associated with deterioration of renal function in IgAN. However, this study was limited to a small cohort of patients with familial IgAN, and it is uncertain at present whether this finding has a broader applicability in sporadic IgAN.

\section{Genetic Influences on Candidate Biomarkers for IgAN}

In IgAN, there continues to be interest in biomarkers, other than conventional measures of renal excretory function and proteinuria, which may have clinical application. The best studied example is the alteration in the $\mathrm{O}$-glycosylation of serum and mesangial IgA, well described in IgAN, and usually regarded as having a significant role in the pathogenesis. Altered $O$-glycosylation of IgA1 was first described in Europeans [29] but has since been identified in all ethnicities studied and has been reported to be heritable, with up to $25 \%$ of healthy blood relatives of subjects with IgAN having abnormal serum IgA1 glycosylation [30]. Abnormal serum IgA1 $O$-glycosylation is typically found in $90 \%$ of patients with IgAN. It is widely considered that the explanation for altered IgA1 glycosylation lies in abnormal function of the specific glycosyltransferases which mediate post-translational glycosylation of IgA1, and given that abnormal serum IgA1 glycosylation is heritable, that the basis for altered function is genetic variation in the genes encoding those enzymes. However, until the publication of GWAS in IgAN, both functional studies of these enzymes and also candidate gene case control studies were notably inconsistent in providing evidence for enzyme dysfunction. For example, there are conflicting data for C1GALT1, the gene encoding the key enzyme $\beta 1,3$ Gal transferase (on chromosome 7p14-p13) and C1GALT1C1 (on chromosome Xq24) encoding its chaperone protein, Cosmc (C1GALT specific molecular chaperone). Whereas in a Chinese population with IgAN three SNPs of C1GALT1 have been reported in significantly different frequency in IgAN and healthy controls without differences in C1GALT1C1 [31], this has not been confirmed in Europeans [32]. 
In GWAS, there has been no association between IgAN and SNPs in regions encoding enzymes and other proteins relevant to IgA1 glycosylation pathways, which is surprising given that hypotheses that were developed before GWAS data became available emphasized a likely central role for genetically determined changes in glycosylation enzymes. The lack thus far of evidence for a genetic defect in post-translational glycosylation machinery means that alternative explanations for altered IgA1 glycosylation in IgAN must be more strongly considered such as a defect in mucosal immunity altering $\operatorname{IgA} 1$ response to mucosal antigen, or the possibility that a mucosal type of IgA1 (which has a different glycosylation pattern to serum IgA1) is reaching the circulation because of altered homing of $\mathrm{T}$ and $\mathrm{B}$ cells with functions relevant to IgA production.

\section{The Contribution of GWAS to Current Understanding of the Pathogenesis of IgAN}

Recent discussions of GWAS findings in IgAN have emphasised that there are risk alleles identified in regions of the genome close to genes with biological plausibility, notably regions coding for HLA and complement. However, language should be used with care; in no case so far have hypotheses emerging from these GWAS findings undergone confirmatory functional testing in vitro or in vivo. In no case yet has there been direct sequencing of genes of interest to confirm that the risk alleles identified by SNP in GWAS are indeed identifying alterations in functional regions of the genes of interest.

Interest in novel hypotheses should also not divert attention from the most striking finding that in all the published GWAS, the risk alleles with the statistically strongest associations are within HLA. The complexity of HLA makes it a challenging region of the genome to study, but work to establish the functional and genetic significance of HLA associations with IgAN should be a priority.

While emphasizing the important new insights which have emerged from GWAS, it should not be forgotten that even the latest and largest GWAS meta-analysis only attributes $7.6 \%$ of disease risk to genetic factors in Chinese, and 6.2\% in Europeans [24].

\section{The Importance of Epigenetics}

The focus of this review is the role played by genetic factors (i.e. changes in DNA sequence) in the pathogenesis and progression of IgAN. However, it is important to remember the likelihood that epigenetic factors, as yet largely uninvestigated, may be equally important. Epigenetic mechanisms are those not involving changes in DNA sequence, but which can alter gene regulation by modulating chromatin access to transcription. The interactions of genetics, epigenetics, and environment provide a much more complex basis for understanding pathogenesis and the emergence of phenotypic variability [33].

Evidence for epigenetic factors in the pathogenesis of IgAN has only begun to emerge in the last few years. Generic processes common to most progressive kidney diseases have been studied - notably epigenetic factors influencing fibrosis, and also, with advanced kidney disease, the epigenetic impact of uraemia itself. But disease-specific evidence is emerging in the role of microRNAs, short non-coding RNAs that regulate gene expression. Preliminary evidence suggests that they may play a role in altered IgA1 glycosylation in IgAN $[34,35]$.

\section{Next Steps}

\section{Should There Be a Further GWAS?}

Available data from GWAS now include cohorts totaling $~ 5,000$ patients with IgAN collected across three continents. Proponents of increasing cohort size will argue that this will allow the identification of a potentially large number of modifier genes with small genetic effect which will all influence the final IgAN phenotypes.

In our view, however, enlarging similar cohorts to identify additional low-risk alleles at this stage will add complexity without clarity. It will be more valuable to work through the hypotheses generated by the now known risk alleles, using direct sequencing and functional studies, to see which hold up under scrutiny.

It may however be valuable to extend GWAS to a wider range of populations within the broad ethnic groups so far analysed: for example, ensuring a wider range of populations with subjects from all parts of Europe as well as populations of European origin who have migrated to North America and Australia. Or widening observations in Asian patients where in the largest GWAS there are only 445 Japanese patients and no Korean patients compared to several thousand Chinese patients.

\section{Improved Phenotyping}

The one justification for additional GWAS would be if 'deep' phenotyping could be achieved in existing or new cohorts. Data on the rate and severity of progressive loss of renal function, data on histopathological variation (us- 
ing the widely accepted Oxford Classification), data on transplant recurrence risk, reliable data on IgAN in family members of probands: all of these would provide a substrate for valuable new analyses, notably to distinguish risk alleles for susceptibility to IgAN from alleles associated with risk of progression. However, such phenotyping can be challenging. In many health economies, patients are referred from wide areas to major centres for specialist assessment including diagnostic renal biopsy. Such centres can therefore in turn recruit significant numbers of patients into GWAS, if inclusion only requires that a diagnosis of IgAN has been made. However, many such patients then return for further clinical followup to a physician who may be far away and less committed to the research, making sequential clinical data collection inconsistent.

\section{Whole-Genome and Exome Sequencing}

At present, it is not clear what impact next-generation sequencing techniques will have on our understanding of the pathogenesis of IgAN. It is almost certain, however, that in the coming years there will be whole-genome and exome sequencing of IgAN cohorts, and these are likely to reveal novel disease-associated alleles. However, as with all genetic techniques, the data generated by nextgeneration sequencing will only be as good as the quality and depth of the phenotyping of the population studied.

\section{Disclosure Statement}

The authors declare no conflict of interest.

\section{References}

1 Boyd JK, Cheung CK, Molyneux K, Feehally J, Barratt J: An update on the pathogenesis and treatment of IgA nephropathy. Kidney Int 2012;81:833-843.

- 2 Waldherr R, Rambausek M, Duncker WD, Ritz E: Frequency of mesangial IgA deposits in a non-selected autopsy series. Nephrol Dial Transplant 1989;4:943-946.

-3 Suzuki K, Honda K, Tanabe K, Toma H, Nihei $\mathrm{H}$, Yamaguchi $\mathrm{Y}$ : Incidence of latent mesangial IgA deposition in renal allograft donors in Japan. Kidney Int 2003;63:2286-2294.

-4 Gutierrez E, Zamora I, Ballarin A, Arce Y, Jimenez S, Quereda C, Olea T, Martinez-Ara J, Segarra A, Bernis C, et al: Long-term outcomes of IgA nephropathy presenting with minimal or no proteinuria. J Am Soc Nephrol 2012;23:1753-1760.

5 Berthoux FC, Laurent B, Alamartine E, Diab $\mathrm{N}$ : New subgroup of primary IgA nephritis with thin glomerular basement membrane (GBM): syndrome or association. Nephrol Dial Transplant 1996;11:558-559.

6 Berger J, Hinglais N: Intercapillary deposits of IgA-IgG (in French). J Urol Nephrol (Paris) 1968;74:694-695.

-7 Prakash S, Kanjanabuch T, Austin PC, Croxford R, Hsu CY, Choi AI, Cattran DC: Continental variations in IgA nephropathy among Asians. Clin Nephrol 2008;70:377-384.

-8 Kiryluk K, Li Y, Sanna-Cherchi S, Rohanizadegan M, Suzuki H, Eitner F, Snyder HJ, Choi M, Hou P, Scolari F, et al: Geographic differences in genetic susceptibility to IgA nephropathy: GWAS replication study and geospatial risk analysis. PLoS Genet 2012;8: e1002765.

-9 Berger J, Levy M: Epidemiology of idiopathic IgA nephropathy. Semin Nephrol 1987;7: 277-279.
10 McQuarrie EP, Mackinnon B, Young B, Yeoman L, Stewart G, Fleming S, Robertson S, Simpson K, Fox J, Geddes CC: Centre variation in incidence, indication and diagnosis of adult native renal biopsy in Scotland. Nephrol Dial Transplant 2009;24:1524-1528.

-11 Schena FP, Scivittaro V, Ranieri E, Sinico R, Benuzzi S, Di Cillo M, Aventaggiato L: Abnormalities of the IgA immune system in members of unrelated pedigrees from patients with IgA nephropathy. Clin Exp Immunol 1993;92:139-144.

12 Schena FP, Cerullo G, Rossini M, Lanzilotta SG, D’Altri C, Manno C: Increased risk of end-stage renal disease in familial IgA nephropathy. J Am Soc Nephrol 2002,13:453460.

13 Gharavi AG, Yan Y, Scolari F, Schena FP, Frasca GM, Ghiggeri GM, Cooper K, Amoroso A, Viola BF, Battini G, et al: IgA nephropathy, the most common cause of glomerulonephritis, is linked to 6q22-23. Nat Genet 2000;26: 354-357.

14 Bisceglia L, Cerullo G, Forabosco P, Torres DD, Scolari F, Di Perna M, Foramitti M, Amoroso A, Bertok S, Floege J, et al: Genetic heterogeneity in Italian families with IgA nephropathy: suggestive linkage for two novel IgA nephropathy loci. Am J Hum Genet 2006; 79:1130-1134.

15 Paterson AD, Liu XQ, Wang K, Magistroni R, Song X, Kappel J, Klassen J, Cattran D, St George-Hyslop P, Pei Y: Genome-wide linkage scan of a large family with IgA nephropathy localizes a novel susceptibility locus to chromosome 2q36. J Am Soc Nephrol 2007; 18:2408-2415.

16 Karnib HH, Sanna-Cherchi S, Zalloua PA, Medawar W, D'Agati VD, Lifton RP, Badr K, Gharavi AG: Characterization of a large Leb- anese family segregating IgA nephropathy. Nephrol Dial Transplant 2007;22:772-777.

17 Deltas C, Pierides A, Voskarides K: Molecular genetics of familial hematuric diseases. Nephrol Dial Transplant 2013;28:2946-2960.

-18 SI Hsu, SB Ramirez, MP Winn, JV Bonventre, WF Owen: Evidence for genetic factors in the development and progression of IgA nephropathy. Kidney Int 2000;57:1818-1835.

19 Tishkoff SA, Verrelli BC: Role of evolutionary history on haplotype block structure in the human genome: implications for disease mapping. Curr Opin Genet Dev 2003;13:569575 .

20 MR van der Sijde, Ng A, Fu J: Systems genetics: from GWAS to disease pathways. Biochim Biophys Acta 2014;1842:1903-1909.

21 Feehally J, Farrall M, Boland A, Gale DP, Gut I, Heath S, Kumar A, Peden JF, Maxwell PH, Morris DL, et al: HLA has strongest association with IgA nephropathy in genome-wide analysis. J Am Soc Nephrol 2010;21:17911797.

22 Gharavi AG, Kiryluk K, Choi M, Li Y, Hou P, Xie J, Sanna-Cherchi S, Men CJ, Julian BA, Wyatt RJ, et al: Genome-wide association study identifies susceptibility loci for IgA nephropathy. Nat Genet 2011;43:321-327.

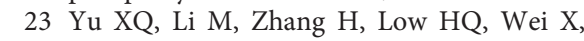
Wang JQ, Sun LD, Sim KS, Li Y, Foo JN, et al: A genome-wide association study in Han Chinese identifies multiple susceptibility loci for IgA nephropathy. Nat Genet 2012;44: 178-182.

24 Kiryluk K, Li Y, Scolari F, Sanna-Cherchi S, Choi M, Verbitsky M, Fasel D, Lata S, Prakash S, Shapiro S, et al: Discovery of new risk loci for IgA nephropathy implicates genes involved in immunity against intestinal pathogens. Nat Genet 2014;46:1187-1196. 
25 Roos A, Rastaldi MP, Calvaresi N, Oortwijn BD, Schlagwein N, van Gijlswijk-Janssen DJ, Stahl GL, Matsushita M, Fujita T, van Kooten C, et al: Glomerular activation of the lectin pathway of complement in IgA nephropathy is associated with more severe renal disease. J Am Soc Nephrol 2006; 17: 1724-1734.

-26 Sebat J, Lakshmi B, Troge J, Alexander J, Young J, Lundin P, Maner S, Massa H, Walker M, Chi M, et al: Large-scale copy number polymorphism in the human genome. Science 2004;305:525-528.

-27 Aitman TJ, Dong, Vyse TJ, Norsworthy PJ, Johnson MD, Smith J, Mangion J, RobertonLowe C, Marshall AJ, Petretto E, et al: Copy number polymorphism in Fcgr3 predisposes to glomerulonephritis in rats and humans. Nature 2006;439:851-855.
28 Sallustio F, Cox SN, Serino G, Curci C, Pesce F, De Palma G, Papagianni A, Kirmizis D, Falchi M, Schena FP: Genome-wide scan identifies a copy number variable region at $3 p 21.1$ that influences the TLR9 expression levels in IgA nephropathy patients. Eur J Hum Genet 2014, Epub ahead of print.

29 Allen AC, Harper SJ, Feehally J: Galactosylation of $\mathrm{N}$ - and O-linked carbohydrate moieties of IgA1 and IgG in IgA nephropathy. Clin Exp Immunol 1995;100:470-474.

30 Hastings MC, Moldoveanu Z, Julian BA, Novak J, Sanders JT, McGlothan KR, Gharavi AG, Wyatt RJ: Galactose-deficient IgA1 in African Americans with IgA nephropathy: serum levels and heritability. Clin J Am Soc Nephrol 2010;5:2069-2074.

31 Li GS, Zhang H, Lv JC, Shen Y, Wang HY: Variants of C1GALT1 gene are associated with the genetic susceptibility to IgA nephropathy. Kidney Int 2007;71:448-453.

32 Malycha F, Eggermann T, Hristov M, Schena FP, Mertens PR, Zerres K, Floege J, Eitner F: No evidence for a role of cosmc-chaperone mutations in European IgA nephropathy patients. Nephrol Dial Transplant 2009;24:321324.

33 Dwivedi RS, Herman JG, McCaffrey TA, Raj DS: Beyond genetics: epigenetic code in chronic kidney disease. Kidney Int 2011;79: 23-32.

34 Serino G, Sallustio F, Cox SN, Pesce F, Schena FP: Abnormal miR-148b expression promotes aberrant glycosylation of IgA1 in IgA nephropathy. J Am Soc Nephrol 2012;23: 814-824.

35 Szeto CC, Li PK: MicroRNAs in IgA nephropathy. Nat Rev Nephrol 2014;10:249-256. 